\title{
Medical Toxicology and Public Health: Update on Research and Activities at the Centers for Disease Control and Prevention
}

\author{
Manish Patel, $M D^{\mathrm{a}}$, Martin Belson, $M D^{\mathrm{a}}$, John Osterloh, $M D^{\mathrm{b}}$, Michael Schwartz, $M D^{\mathrm{c}}$
}

\author{
a Division of Environmental Hazards and Health Effects, National Center for Environmental Health \\ b Division of Laboratory Sciences, National Center for Environmental Health \\ c Division of Toxicology, Agency for Toxic Substances and Disease Registry
}

\section{Division of Environmental Hazards and Health Effects, Health Studies Branch (NCEH, CDC)}

Children's Potential Exposure to Arsenic from CCA-Treated Wooden Playground Structures: A Pilot Study ${ }^{1}$

The magnitude of the arsenic dose a child may receive from playing on or under a CCA-treated structure is unknown. Further research is needed to determine the body burden of arsenic in children who play around and on CCA-treated wood. Our goals are to (1) provide preliminary data on the human pharmacokinetics of arsenic from CCA-treated wood exposure and (2) to guide the development of a larger, future study that will evaluate the potential long-term health risks of arsenic exposure from routine play on CCA-treated structures.

\section{An Assessment of Environmental Contamination from Chemicals Used and Produced during Methamphetamine Manufacture: A Pilot Study ${ }^{1}$}

Little is known about the health effects from chronic exposure to residual chemicals that may persist after remediation of residential methamphetamine labs. We are in the process of performing an environmental assessment of these properties to understand what residual chemicals new occupants may be exposed to. This study will examine whether residential methamphetamine labs still contain contaminants after remediation that may pose a health risk to residents. Information about chemicals that persist after remediation will be useful to guide cleanup efforts, as well as to assist in the development of a broader, future study. A future study would attempt to determine whether there is an association between chronic exposure to residual chemicals and adverse health effects among residents of residential methamphetamine labs that have gone through remediation.

\section{Persistent Organic Pollutants (POPS) in Alaska ${ }^{1}$}

The Health Studies Branch is monitoring selected persistent organic pollutants (POPs) and heavy metals in umbilical cord blood and maternal blood in Alaskan Natives. The POPs levels in these samples will be related to pregnancy outcomes (e.g., fullterm live birth, miscarriage, birth defects) and to the rate of infectious diseases in the first year of life of infants. To date, more than 200 women and 90 infants from Bethel, Barrow, and the Aleutian and Pribilof Islands have been enrolled in the study.

\section{Division of Laboratory Sciences (NCEH, CDC)}

National Report on Human Exposure to Environmental Chemicals ${ }^{2}$

The 3rd National Report on Human Exposure to Environmental Chemicals is accessible at: www.cdc.gov/exposurereport. The Report provides biomonitoring exposure data for a representative sampling of the U.S. population. Medical toxicologists are integrating the statistical analysis with written text about each of the 148 chemicals measured in blood and urine by the Division of Laboratory Sciences.

Editor's Note: The following are areas of research and activity in which medical toxicologists are actively involved at the National Center for Environmental Health and Agency for Toxic Substances and Disease Registry (NCEH/ATSDR) at the Centers for Disease Control and Prevention. The Journal of Medical Toxicology will periodically highlight some of these activities to illustrate the growing relationship between medical toxicology and public health. 
Ongoing Collaboration and Research in Chemical Terrorism Associated Issues ${ }^{2}$

Medical toxicologists in the Division of Laboratory Sciences are collaborating on a number of interfederal agency activities that include: testing for chemical warfare agents (CWA) in military workers, consideration of the public availability of CWA antidotes, and the development of new biotoxin assays for human specimens.

\section{Division of Toxicology, Emergency Response and Scientific Assessment Branch (ATSDR, CDC)}

Medically-Oriented Fact Sheets (MOFS) ${ }^{3}$

Medical toxicologists within the Division of Toxicology are currently involved in creating a series of Medically-Oriented Fact
Sheets (MOFS) for physicians and first responders that describe the recognition, initial management, diagnosis and treatment of chemically exposed patients. This series of single page, acute management instructions-companion to the much longer Medical Management Guidelines for Acute Chemical Exposures (MMGs) is designed for emergency and primary care physicians. 